\title{
Long-term safety of statin-fibrate combination treatment in the management of hypercholesterolaemia in patients with coronary artery disease
}

\author{
M D Feher, J Foxton, D Banks, A F Lant, R Wray
}

\begin{abstract}
Objective-To evaluate the long-term safety profile of treatment with a statinfibrate combination in a cohort of patients with documented coronary artery disease.
\end{abstract}

Design-Retrospective cohort analytical study.

Setting-District general hospital.

Patients-102 (81 male and 21 female) hypercholesterolaemic (total cholesterol concentration $>6.5 \mathrm{mmol} / \mathrm{l}$ ) patients with documented coronary artery disease and who had been treated with a statin-fibrate combination for over 1 year. Coronary artery disease was confirmed by angiography in 93 patients and by a positive (Bruce protocol) exercise test in the remainder. Fifty eight patients had a history of previous coronary bypass graft surgery.

Interventions-Twice daily lipid lowering treatment was given, with the fibrate administered in the morning (either bezafibrate $400 \mathrm{mg} \quad(\mathrm{n}=101)$ or fenofibrate $200 \mathrm{mg}(n=1))$ and the statin in the evening (either simvastatin $10 \mathrm{mg}(\mathrm{n}=23), 20 \mathrm{mg}(\mathrm{n}=72)$, or $40 \mathrm{mg}(\mathrm{n}=2)$ or pravastatin $10 \mathrm{mg}$ $(\mathrm{n}=1)$ or $20 \mathrm{mg}(\mathrm{n}=4))$. Treatment continued for $1(n=9), 2(n=58)$, or 3 $(\mathbf{n}=35)$ years.

Main outcome measures-Selected laboratory variables (total cholesterol concentration and liver (aspartate transaminase (AST)) and muscle enzyme (creatine kinase (CK)) activities) and documented symptomatology.

Clinical

Pharmacology and

Therapeutics, Charing

Cross and

Westminster Medical

School, London

M D Feher

J Foxton

A F Lant

Department of

Cardiology, Conquest

Hospital, Hastings

D Banks

R Wray

Correspondence to:

Dr M Feher, Department of

Clinical Pharmacology and

Therapeutics, Charing

Therapeutics, Charing

Medical School, 369

Fulham Road, London

SWlham Road $10 \mathrm{NH}$.

SW $109 \mathrm{NH}$.

14 February 1995
Results-A mean (SD) total cholesterol concentration of $5 \cdot 2 \quad(0.8) \mathrm{mmol} / 1$ was achieved after combined treatment for 1 year which was maintained at annual follow up. Over a maximum 3 year follow up no patient reported myalgic symptoms and none had a measured CK activity $>10$ times above nomal. Four men on a simvastatinbezafibrate combination had a CK activity rise to less than three times normal. Fourteen patients with a negative history of alcohol excess (consumption < 21 units/week) had borderline raised AST values.

Conclusions-Statin-fibrate combination treatment for up to 3 years in a cohort of patients with coronary $\vec{D}$ artery disease was not associated with serious disturbances in biochemical markers of muscle or liver function.

(Br Heart f 1995;74:14-17)

Keywords: statin-fibrate combination therapy; hypercholesterolaemia; coronary artery disease

Increasing evidence from secondary prevention trials confirms that effective cholesterol lowering retards progression of coronary atherosclerosis in patients with established coronary artery disease. ${ }^{12}$ Lower cholesterol concentrations are consequently considered desirable in these patients to reduce the exaggerated risk of subsequent coronary events. ${ }^{23}$ Combination treatment with different hypolipidaemic drug groups has been shown to enhance cholesterol lowering ${ }^{45}$ and may have a useful role in achieving this therapeutic target in such high risk patients.

The 3-hydroxy-3-methylglutaryl-coenzyme A reductase inhibitor or statin group of drugs are the most potent hypocholesterolaemic agents currently available and are generally well tolerated. ${ }^{46}$ Complications reported with statin treatment include increases in muscle and liver enzyme activities, with rare individual cases of myositis (defined as creatine kinase (CK) activity $>10$ times above normal) and rhabdomyolysis when statins have been given in combination with other drugs $N$ including cyclosporin and the fibric acid $N$ derivative gemfibrozil. ${ }^{78}$ The potential haz- N ards reported in these individual cases have had a negative influence on the widespread use of statin in combination with other fibric acid derivatives. The aim of this study was to evaluate the long-term safety profile of treatment with a statin-fibrate combination in a cohort of patients with documented coronary artery disease.

Patients and methods

A total of 102 (81 male and 21 female) hypercholesterolaemic (defined as initial total cholesterol concentration $>6.5 \mathrm{mmol} / \mathrm{l}$ ) patients with documented coronary artery disease and who had been treated with a statin-fibrate combination for over 1 year were studied. Twice daily treatment was given, with the fibrate 
Table 1 Details of four male patients with an increase in creatine kinase (CK) above normal range $(<374$ UIl)

\begin{tabular}{lll}
\hline & $\begin{array}{c}\text { Dosage } \\
(\mathrm{mg})\end{array}$ & No of patients \\
\hline Lipid lowering treatment & & \\
$\quad$ Bezafibrate & 400 & 4 \\
plus & 20 & 2 \\
simvastatin & 10 & 2 \\
or simvastatin & & 3 \\
Other treatment & & 2 \\
$\quad$ Aspirin & 2 \\
$\quad$ NSAI agent & & \\
$\quad$ Diuretics & & 0 \\
Patient characteristics & & 1 \\
$\quad$ Alcohol intake & \\
$\quad>21$ units/week) & & 0 \\
$\quad$ Current smoker & & 1 (CK 958 U/l) \\
Renal failure & & 0 \\
Event & & \\
$\quad$ CK $>2$ times upper normal & & \\
Myalgia & &
\end{tabular}

administered in the morning (either bezafibrate $400 \mathrm{mg}(\mathrm{n}=101)$ or fenofibrate $200 \mathrm{mg}(\mathrm{n}=$ 1)) and the statin in the evening (either simvastatin $10 \mathrm{mg}(\mathrm{n}=23), 20 \mathrm{mg}(\mathrm{n}=72)$, or $40 \mathrm{mg}$ $(n=2)$ or pravastatin $10 \mathrm{mg}(\mathrm{n}=1)$ or $20 \mathrm{mg}$ $(n=4))$.

Coronary artery disease was confirmed by angiography in 93 patients and by a positive (Bruce protocol) exercise test in the remainder. Fifty eight patients (57\%) had a history of previous coronary bypass graft surgery. Seventeen patients (17\%) were current smokers. No patient had received an organ transplant or was receiving cyclosporin. In addition to routine clinic supervision, all patients were advised to contact the clinic nurse or doctor directly by telephone if symptoms developed, in particular myalgia. This report describes only the selected laboratory variables recorded for all patients in addition to the documented symptomatology. The biochemical variables measured by standard automated procedures included total cholesterol concentration, liver (aspartate transaminase (AST)) and muscle enzyme (CK) activities, together with the clinical symptoms recorded for each patient during their routine clinic attendance.

Table 2 Alcohol consumption and plasma creatine kinase (CK) activities in 14 patients treated with statin-fibrate combination treatment who developed plasma activities of AST $>40$ U/l

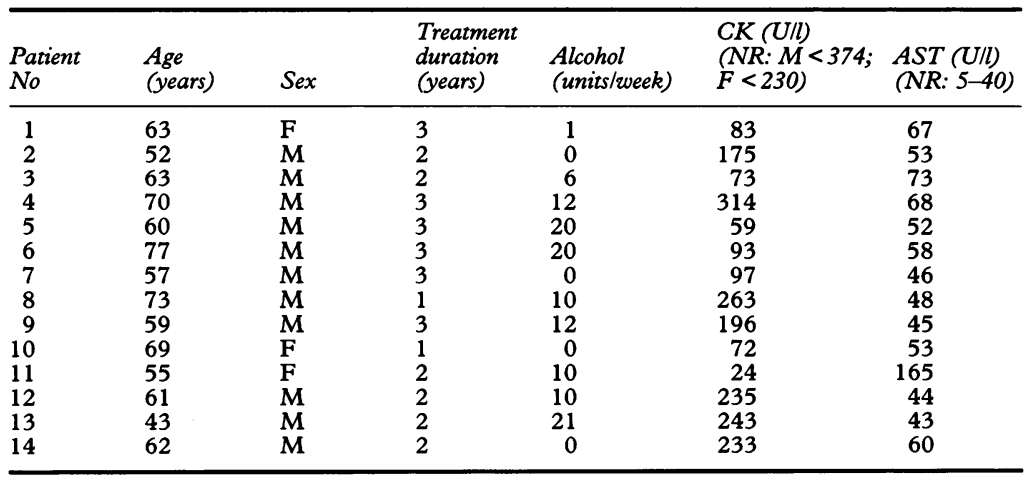

NR, normal range.

\section{Results}

Of the cohort of 102 patients, safety data were collected prospectively over a 3 year treatment period in 35, over 2 years in 58 and over 1 year in nine. For the whole group, the pretreatment mean (SD) serum total cholesterol concentration of $7.4(1.6) \mathrm{mmol} / 1$ was reduced to $5.2(0.8) \mathrm{mmol} / \mathrm{l}$ after combination drug treatment for 1 year. This reduction in total cholesterol was maintained at the 2 and 3 year follow up.

Over a maximum 3 year follow up no patient reported myalgic symptoms and none had increased plasma CK activity 10 times above normal. Four male patients treated with a simvastatin-bezafibrate combination had an increase in $\mathrm{CK}$ activity above the reference range (table 1). Peak $\mathrm{CK}$ values were observed in two of these patients (958 and $618 \mathrm{U} / \mathrm{l})$ after treatment for 1 year, in the third patient at 2 years $(418 \mathrm{U} / \mathrm{l})$ and in the fourth at 3 years $(451 \mathrm{U} / 1)$. Fourteen patients despite a negative history of alcohol excess (consumption < 21 units/week) had borderline raised AST values but none had significant disturbances in CK activity (table 2).

\section{Discussion}

Statin drugs are widely prescribed for the treatment of hypercholesterolaemia due to their established cholesterol lowering efficacy and tolerability. However, information from long-term usage in prospective randomised trials of statins is only just emerging. ${ }^{2}$ Simvastatin has been shown to achieve $70 \%$ of its lipid lowering effect at low dose, ${ }^{9}$ hence a further increase in dosage may not enhance substantially cholesterol lowering, but may be associated with the hazard of dose dependent adverse effects.

Current consensus guidelines in Britain recommend a desirable cholesterol concentration of $<5.2 \mathrm{mmol} / \mathrm{l}$ in patients at highest risk of vascular disease, in particular those with established coronary artery disease. ${ }^{3}$ The use of combination lipid lowering treatment is often required to achieve this goal, and has clearly been shown to enhance cholesterol lowering. ${ }^{45}$ Most regimens have excluded the combination of a fibrate with a statin due to individual case reports of muscle toxicity. ${ }^{78}$ Nicotinic acid derivatives or bile acid sequestrants have been the main choice as partners to statins in combination treatment but widespread use is often limited because of adverse gastrointestinal effects particularly for the bile acid sequestrants, while rash, flushing and rarely myopathy have been reported with nicotinic acid. ${ }^{10}$ Additionally, hypertriglyceridaemia is a well documented side effect of bile acid sequestrants ${ }^{11}$ which would mitigate their inclusion in the management of patients with a mixed hyperlipidaemia.

Reports of greatly increased plasma CK activity and rhabdomyolysis with statin treatment have included patients who have been organ transplant recipients necessitating the co-administration of the immunosuppressive agent cyclosporin. ${ }^{12-14}$ There is clear 
evidence of a pharmacokinetic interaction with this drug combination resulting in a fivefold increase in circulating statin concentrations. ${ }^{15}$ However a profound rise in $\mathrm{CK}$ values with lovastatin treatment has been observed rarely in the absence of immunosuppressive treatment. ${ }^{16}$ This finding has also been reported for lovastatin given with either nicotinic acid ${ }^{10}$ or gemfibrozil. ${ }^{78}$ In most cases where there were clinical features of myositis or rhabdomyolysis, lovastatin had been the prescribed statin. By comparison, there are no cases using other statins and there has been only one report of an association with low dose simvastatin, with the adverse event occurring 3 months after the start of treatment. ${ }^{17}$

The recent evidence that untreated hypercholesterolaemic patients may have increased muscle enzyme activities, ${ }^{18}$ both before and after exercise (JWA Smit et al, 62nd European Atherosclerosis Society meeting, Jerusalem, Israel, 1993) highlights the difficulties in attributing a single causative role to statin or fibrate drugs (either singularly or in combination) in initiating the altered muscle biochemistry.

There have been reports of transient increases in serum liver transaminases with fibrate treatment ${ }^{1920}$ and up to three times normal values in about $1.5 \%$ of patients treated with statins. ${ }^{21}$ There are few data, however, on the numbers of patients withdrawn from such treatment because of this adverse effect. It is of interest in the present study in which both drug groups were used in combination that only one patient had an increase of three times the upper normal AST activity and the remaining patients had only borderline changes in AST values.

Cholesterol lowering effects of combined statin-fibrate treatment in different types of hyperlipidaemia have been previously studied (H. Vanhanen et al, international symposium on atherosclerosis, Illinois, United States, 1991 ; K. Takata et al, 11 th international symposium on drugs affecting lipid metabolism, Florence, Italy, 1992; R. Volpe et al, 62nd European Atherosclerosis Society meeting on familial hypercholesterolaemia, Jerusalem, Israel, 1993). ${ }^{22-31}$ Most patients had been treated for less than 10 months, $(\mathrm{H}$ Vanhanen et al, international symposium on atherosclerosis; K Takata et al, 11th international symposium on drugs affecting lipid metabolism, Florence, Italy, 1992; R Volpe et al, 62nd European Atherosclerosis Society meeting on familial hypercholesterolaemia, Jerusalem, Israel, 1993), ${ }^{22-29}$ while two reports included patients treated for up to 18 months. ${ }^{3031}$ No study reported exclusively on patients with documented coronary artery disease. These short-term studies in patients without confirmed coronary disease but who had either hypercholesterolaemia or a mixed hyperlipidaemia used treatment combinations of clofibrate, ${ }^{22}$ bezafibrate (K Takata et al, 11 th international symposium on drugs affecting lipid metabolism, Florence, Italy, 1992), ${ }^{23-262830}$ gemfibrozil (H Vanhanen et al, international symposium on atherosclerosis; R. Volpe et al, 62nd European Atherosclerosis Society meeting on familial hypercholesterolaemia, Jerusalem, Israel, 1993), ${ }^{2731}$ or fenofibrate $^{29}$ as the fibrate addition to the statin and lovastatin, ${ }^{22} 25$ simvastatin, ${ }^{24}{ }^{26-31}$ fluvastatin $^{28}$ or pravastatin as the chosen statin (R. Volpe et al, 62nd European Atherosclerosis Society meeting on familial hypercholesterolaemia, Jerusalem, Israel, 1993)..$^{2324} \mathrm{It}$ is of interest that none of these studies documented any occurrence of myositis or myopathy.

While previous individual case reports have given concern about the concurrent use of a statin with a fibrate in particular gemfibrozil with lovastatin, ${ }^{478}$ this safety study involving patients with coronary artery disease has confirmed the findings of previous studies of statin-fibrate treatment which were of shorter duration and involved fewer numbers of patients. The combination was not only effective in achieving the desirable cholesterol concentration in a large number of patients at high risk of vascular disease, but was also associated with no serious disturbance in biochemical markers of muscle or liver function.

Our observations may reflect the particular selection of treated individuals who were regular clinic attendees, tablet compliant, avoided alcohol excess and who had not been a transplant recipient requiring cyclosporin or was in established renal failure. The use of fibrates-other than gemfibrozil-together with statins - other than lovastatin - as well as the intensive monitoring through regular clinic attendance with telephone back up, may have also accounted for the observed lack of any serious adverse event in this study.

Combination treatment of selected statin and fibrate drugs with appropriate monitoring may have a useful role in achieving desirable cholesterol concentrations, without hazard, in patients with documented coronary artery disease.

1 Rossouw JE, Lewis B, Rifkind BM. The value of lowering cholesterol after myocardial infarction. $N$ Engl f Med 1990;323:1112-9.

2 Scandinavian Simvastatin Survival Study Group. Randomised trial of cholesterol lowering in 4444 patients with coronary heart disease: the Scandinavian simvastatin survival study (4S). Lancet 1994;344: 1383-9.

3 Betteridge DJ, Dodson PM, Durrington PN, et al. Management of hyperlipidaemia: guidelines of the British Hyperlipidaemia Association. Postgrad Med $f$ 1993;69:359-69.

4 Grundy SM. HMG-Co A Reductase Inhibitors for treatment of hypercholesterolaemia. N Engl f Med 1988;319 (1):24-33.

5 East C, Bilheimer DW, Grundy SM. Combination drug therapy for familial combined hyperlipidaemia. Ann Intern Med 1988;109:25-32.

6 Maher VM, Thompson GR. HMG CoA reductase inhibitors as lipid-lowering agents: five years experience with lovastatin and an appraisal of simvastatin and pravastatin. $Q 7 \mathrm{Med} 1990 ; 74: 165-75$.

7 Marais GE, Larson K. Rhabdomyolysis and acute renal failure induced by combination lovastatin and gemfibrozil therapy. Ann Intern Med 1990;112(3): 228-30.

8 Pierce LR, Wysowki DK, Gross TP. Myopathy and rhabdomyolysis associated with lovastatin-gemfibrozil combidomyolysis associated with lovastatin-gem
nation therapy. $¥ A M A 1990 ; 264: 71-5$.

9 Marshall S, Elliott HL, Meredith PA. A population approach to dose versus response relationships for simvastatin in hypertensive hypercholesterolaemic patients. Br $\mathcal{F}$ Clin Pharmacol 1994;37(5):494P.

10 Reavan P, Witztum J. Lovastatin, nicotinic acid and rhabdomyolysis. Ann Intern Med 1988;109:597-8. 
11 Crouse JR III. Hypertriglyceridaemia: a contraindication to the use of bile acid binding resins. $A m \mathcal{F}$ Med 1987;83:243-8.

12 Norman DJ, Illingworth DR, Munson J, Hosenpud J. Myolysis and acute renal failure in a heart-transplant patient receiving lovastatin. $N$ Engl $f$ Med 1988; 318:46-7.

13 East C, Alivizatos PA, Grundy SM, Jones PH, Farmer JA. Rhabdomyolysis in patients receiving lovastatin afte cardiac transplantation. N Engl 7 Med 1988;318:47-8.

14 Corpier CL, Jones PH, Suki WN, et al. Rhabdomyolysis and renal injury with lovastatin use. Report of two cases in cardiac transplant recipients. $\mathscr{F} A M A$ 1988; 260:239-41.

15 Smith PF, Eydelloth RS, Grossman SJ, et al. HMG CoA reductase inhibitor-induced myopathy in the rat cyclosporine $\mathrm{A}$ interaction and mechanism studies. $\mathcal{F}$ Pharmacol Exp Ther 1991;257:1225-35.

16 Ayanian JZ, Fuchs CS, Stone RM. Lovastatin and rhabdomyolysis. Ann Intern Med 1988;109:682-3.

17 Berland Y, Vacher Coponat H, Baz M, Laugier R, Musso JL. Rhabdomyolysis with simvastatin. Nephron 1991; 57:365-6.

18 Bhatnagar D, Durrington PN, Neary R, Miller JP. Elevation of skeletal muscle isoform of serum creatine Elevation of skeletal muscle isoform of serum creatine
kinase in heterozygous familial hypercholesterolaemia. $\mathcal{f}$ Intern Med 1990;228:493-5.

19 Monk JP, Todd PT. Bezafibrate-a review of its pharmacodynamic and pharmakinetic properties, and therapeutic use in hyperlipidaemia. Drugs 1987;33:539-76.

20 Blane GF. Review of European Clinical Experience with Fenofibrate. Cardiology 1989;76(suppl 1):1-13

21 Todd PA, Goa KL. Simvastatin. A review of its pharmacological properties and therapeutic potential in hypercholesterolaemia. Drugs 1990;40:583-607.

22 Illingworth DR, O'Malley IP. The hypolipidaemic effects of lovastatin and clofibrate alone and in combination in patients with type III hyperlipoproteinaemia. Metabolism 1990;39(4):403-9.

23 Lintott CJ, Scott RS, Sharpe DN, et al. Treatment of primary hypercholesterolaemia with simvastatin. Med $f$ Aust 1991;155:433-6.

24 Horsmans Y, Desager JP, Harvengt C. Effects of combined bezafibrate-simvastatin appraised in healthy subjects. F Clin Pharmacol 1992;32:422-6.

25 Yeshurun D, Abukarshin R, Elias N, Lanir A, Naschitz JE. Treatment of severe, resistant familial combined hyperlipidaemia with a bezafibrate-lovastatin combination. Clin Ther 1993;15:355-63.

26 Kehely A, Macmahon M, Wray R, et al. Bezafibrate and simvastatin: an effective and safe combination to treat mixed hyperlipidaemia. Clin Sci 1993;85(suppl 29):68.

27 Wiklund O, Bergman M, Bondjers G, et al. Pravastatin and gemfibrozil alone and in combination for the treatment of hypercholesterolaemia. Am $f$ Med 1993; 94:13-24.

28 Leitersdorf E, Muratti EN, Eliav O, et al. Efficacy and safety of a combination fluvastatin-bezafibrate treatment for familial hypercholesterolaemia; comparative analysis to fluvastatin-cholestyramine combination. $A m \mathcal{F} \mathrm{Med}$ 1994;96:401-7.

29 Weisweiler P. Simvastatin in combination with other lipid lowering drugs (colestipol and fenofibrate): Effects on lowering drugs (colestipol and fenofibrate): Effects on serum lipoproteins. In: Crepaldi G, Tiengo A, Enzi G, eds. Diabetes, obesity and hyperlipidemias IV 1990.

30 Deslypere JP. Addition of fibrates to simvastatin therap in hyperlipidaemic patients. Atherosclerosis 1992;97: 567-71.

31 Da Col PG, Fonda M, Fisicero M, et al. Tolerability and efficacy of combination therapy with simvastatin plus gemfibrozil in type IIb refractory familial combined hyperlipidaemia. Current Therapeutic Research 1993;53: 473-83. 